\title{
Gouvernance et réformes éducatives en Argentine
}

\section{Claudio Suasnábar et Laura Inés Rovelli}

Traducteur : Philippe Rabaté

\section{(2) OpenEdition}

\section{Journals}

Édition électronique

URL : http://journals.openedition.org/ries/868

DOI : $10.4000 /$ ries.868

ISSN : 2261-4265

Éditeur

Centre international d'études pédagogiques

Édition imprimée

Date de publication : 1 décembre 2010

Pagination : 17-26

ISBN : 978-2-8542-583-1

ISSN : $1254-4590$

\section{Référence électronique}

Claudio Suasnábar et Laura Inés Rovelli, «Gouvernance et réformes éducatives en Argentine », Revue internationale d'éducation de Sèvres [En ligne], 55 | décembre 2010, mis en ligne le 01 décembre 2013, consulté le 19 avril 2019. URL : http://journals.openedition.org/ries/868 ; DOI : 10.4000/ries.868

Ce document a été généré automatiquement le 19 avril 2019

(c) Tous droits réservés 


\title{
Gouvernance et réformes éducatives en Argentine
}

\author{
Claudio Suasnábar et Laura Inés Rovelli \\ Traduction : Philippe Rabaté
}

\section{NOTE DE L'ÉDITEUR}

Traduit de l'espagnol

\section{Formation, essor et reconversion du système éducatif argentin}

1 L'un des traits distinctifs de l'éducation en Argentine est la constitution précoce de son système éducatif. Vers la fin du XIXe siècle, l'élite politique, résolument modernisatrice, conçut l'école primaire comme un lieu privilégié pour former les citoyens à être des sujets actifs et impliqués dans leur propre gouvernance (Rose, 1997) et pour faire naître, à travers la transmission de normes, de valeurs et de croyances communes, un sentiment d'appartenance chez une population socialement et culturellement hétérogène. La création de l'appareil d'État s'accompagna de la naissance de corps administratifs et bureaucratiques capables d'assumer les différentes fonctions d'un système de domination moderne. L'école secondaire constitua une pièce maîtresse de cette entreprise et fut également une instance de sélection sociale qui rendit possible l'accès à l'université, lieu réservé à la formation des élites.

2 La structure du système éducatif reproduisit, de manière générale, le modèle européen organisé en niveaux selon des secteurs sociaux et des fonctions différenciés. Malgré cela, et à la différence des expériences européennes, la définition d'une école primaire unique pour toute la population et la hausse accélérée du niveau engendrèrent une dynamique de pression ascendante sur l'école secondaire, dont l'expression principale fut la 
formation d'une classe moyenne. Un autre trait essentiel fut le caractère central de l'État national, qui devint l'acteur principal auquel incombaient non seulement la responsabilité de soutenir et de garantir le service éducatif mais surtout la définition des contenus à transmettre. La promulgation de la loi 1420, en 1884, marqua en ce sens le triomphe des secteurs libéraux, dans la controverse qui les opposait aux catholiques sur la définition et la nature du contrôle de l'éducation (Tedesco, 1986 ; Puiggros, 1990) ${ }^{1}$.

Cet essor du système éducatif se poursuivit et s'approfondit avec l'arrivée du péronisme au gouvernement; ce dernier fut à l'origine d'un processus d'inclusion des secteurs populaires dans de nouveaux circuits éducatifs orientés vers l'éducation pour le travail. À partir des années cinquante et soixante, aux actions éducatives entreprises par l'État national s'ajoutèrent, avec un grand dynamisme, celles - conjointes - des provinces et du secteur privé, ce qui contribua au plus grand essor que le système ait connu de toute son histoire. À son tour, l'apogée des théories du développement conféra à l'état une fonction planificatrice et régulatrice des initiatives privées. Cette redéfinition se concrétisa par la création de divers organismes et secteurs au sein de l'appareil étatique, qui s'efforcèrent d'élaborer les premiers diagnostics socio-éducatifs afin d'articuler l'éducation avec les structures productives. Selon le recensement de 1914, $48 \%$ des enfants âgés de 6 à 14 ans étaient inscrits à l'école primaire, chiffre qui devait atteindre $73,2 \%$ de la population scolaire en 1947. La différenciation progressive du secondaire accompagna cet essor : aux écoles normales et commerciales déjà existantes s'ajouta le développement d'orientations techniques, dans le but de satisfaire les demandes éducatives des secteurs en pleine ascension. De la même manière, l'offre du secteur privé fut concentrée aussi bien à ce niveau qu'à celui de l'enseignement supérieur non universitaire, bien que cette présence ne remette pas en question l'hégémonie publique en matière de prestation de système éducatif. L'Université, pour sa part, connut un processus de massification des inscriptions et de multiplication de ses institutions, provoqué par la nationalisation progressive des universités provinciales et la création de nouvelles institutions publiques. Ces deux processus marquèrent le déclin du modèle d'accès élitiste à l'éducation, qui avait caractérisé la première moitié $\mathrm{du} \mathrm{xx}^{\mathrm{e}}$ siècle, en dépit des éléments de démocratisation présents dans la réforme universitaire de 1918.

4 La diversification de l'offre due à l'émergence de nouveaux prestataires donna naissance à une multiplicité de sous-systèmes superposés géographiquement et entretenant différentes relations de dépendance. Ainsi, dans les provinces qui connaissaient le développement le plus abouti de leur système éducatif, l'essor de l'école primaire et secondaire se fit à travers quatre modalités différentes: des écoles publiques et privées nationales ainsi que des écoles publiques et privées provinciales. Le manque d'articulations horizontales et/ou verticales, auquel s'ajoutèrent les orientations erratiques de la politique éducative, favorisèrent l'apparition d'un conglomérat asystématique d'institutions, caractérisé par une forte désarticulation verticale et aggravé par une diversification des offres de programmes qui confinait à la dispersion. C'est au cours de cette étape que se renforça l'idée de décentraliser l'éducation, sous la forme d'un transfert des institutions scolaires nationales vers les provinciales. Ce processus dura plusieurs décennies et recouvrit différentes significations selon les régimes démocratiques et autoritaires qui s'efforcèrent de l'appliquer ${ }^{2}$.

5 Les longues années de gouvernement militaire consolidèrent des tendances bureaucratiques et autoritaires dans l'administration et la gestion du système éducatif argentin, alors même que sa fonction pédagogique se détériorait, ce qui provoqua une 
perte de qualité et d'excellence des savoirs transmis. Les politiques de coupes des financements nationaux qui accompagnèrent, en règle générale, les cycles de ces régimes, pratiquées dans un contexte où l'éducation poursuivait son développement, finirent par aggraver le diagnostic sur la crise structurelle du système (Braslavsky y Filinus, 1985). Le début d'un nouveau cycle politique dans le pays - celui de la démocratie retrouvée permit la naissance d'un fort consensus social sur la nécessité de transformer le système éducatif, bien que cet accord tarde à prendre la forme concrète d'une proposition de réforme. À l'Université, en dépit du rétablissement d'un schéma normatif pour les différentes maisons d'étude, aucune politique publique active ne fut déployée (Suasnábar, 2005). Dans cette perspective, à la fin des années 1980, les processus économiques d'hyperinflation que connut le pays mirent en évidence la fragilité de l'État et conduisirent les institutions elles-mêmes et leurs acteurs à se charger d'affronter les demandes et les problèmes éducatifs restés en suspens. L'essor du système éducatif accentua, dans ce contexte, les tendances croissantes vers sa différenciation et sa désarticulation (Braslavsky, 1985).

En réponse à la crise, la réforme dite de l'État impliqua une rédéfinition des objectifs de l'intervention étatique dans tous les domaines. À l'approfondissement de la dérégulation de la trame productive qui avait débuté au milieu des années 1970, le second gouvernement de la transition démocratique ajouta un plan de retard dans la répercussion des taux de change et développa un vaste ensemble de réformes centrées sur le processus de privatisation, de dérégulation, de libéralisation commerciale et financière, et de flexibilisation du marché du travail. La redéfinition des règles du jeu entre l'état et la société civile entraîna une restructuration profonde de l'ensemble du système éducatif, en accord avec la progression d'une logique néolibérale présente dans le gouvernement. De telles transformations modifièrent le modèle classique du développement éducatif et les modalités de régulation et de gouvernance qui avaient structuré le système depuis ses origines, malgré la diversité des réformes et changements conjoncturels.

8 L'achèvement du processus de transfert des écoles nationales vers les provinces (exposé dans la Loi de transfert éducatif) visa davantage la réduction des dépenses publiques et la libération de recours financiers pour faire face aux engagements liés à la dette extérieure qu'il n'offrit une réponse aux demandes historiques et légitimes de décentralisation. En 1993, la promulgation de la Loi fédérale d'éducation introduisit un bouleversement de la structure du système : des sept années d'éducation primaire obligatoire et cinq (six, dans certains cas) de secondaire facultative, l'on passa à l'instauration de l'éducation générale basique (EGB). La nouvelle organisation comporta alors trois cycles : une année de niveau initial, neuf années d'éducation de base et trois d'éducation polymodale, avec cinq orientations différenciées et non obligatoires. De ce fait, le caractère obligatoire de l'école fut étendu à dix années mais sans ouvrir l'accès à l'éducation polymodale, ce qui découragea l'ascension des classes populaires dans un système éducatif qui comptait, de manière précoce, un ample champ de couverture pour le niveau primaire.

9 En outre, la réforme entraîna un changement dans la gouvernance de l'éducation, provoqué par la redistribution de fonctions et responsabilités entre les différents agents éducatifs. De la sorte, tandis que le ministère de l'Éducation se réservait la définition et l'élaboration des politiques pour ce secteur ainsi que le suivi de leur application, les provinces se virent confier la gestion effective des services et de leur financement. L'on assista par conséquent au déploiement d'une stratégie à la fois de recentralisation de la 
part de l'État national sur la scène politico-éducative et de reconversion du système, avec l'introduction de nouvelles modalités de régulation et de gestion dirigées vers l'accord sur des politiques et la mise en place d'un dispositif d'information et d'évaluation éducative ; cette évolution introduisit un nouvel univers de valeurs et de critères issus de la culture de l'entreprise (efficience, efficacité et responsabilité sur les résultats). À cet égard, les politiques éducatives, avant d'avoir l'ensemble des citoyens comme principal destinataire, furent orientées vers la « compensation » des inégalités croissantes chez des "populations-objets» considérées comme en danger. Toutefois, la mise en place de politiques focalisées - bien qu'elle soit opératoire selon les critères rationalisateurs et efficientistes des dépenses publiques - ne parvint pas à inverser, mais bien au contraire approfondit la tendance vers la cristallisation de circuits culturels différenciés : aussi déboucha-t-on sur un cercle vicieux de "politiques sociales pauvres" à l'adresse des secteurs populaires.

Sur le plan des politiques d'enseignement supérieur, la période 1989-2000 se caractérisa par la reconfiguration de l'ensemble de ce domaine et par un important interventionnisme étatique (Suasnábar, 2005). La construction d'un certain consensus autour d'un programme de réformes mené par le gouvernement, en accord avec les recommandations de la Banque mondiale, privilégia comme thèmes centraux la qualité, l'évaluation des institutions et la production de ressources propres de la part des universités. La création du secrétariat de Politique universitaire (SPU) et, par la suite, la promulgation de la Loi d'enseignement supérieur (LES) marquèrent le passage d'un type de gouvernement qui combinait des mécanismes d'autorégulation - comme le Conseil interuniversitaire national (CIN) - et une tutelle étatique basée sur le paradigme bienveillant du financement à un modèle dont le trait dominant fut le développement et la différenciation de plusieurs agences de gouvernance et de régulation de l'enseignement supérieur ${ }^{3}$. Le changement dans les formes de financement, à travers des mécanismes compétitifs de distribution, s'instaura de manière parallèle au poids croissant de la SPU dans la définition et la mise en œuvre de programmes spéciaux : ceuxci formèrent le principal outil pour le développement et l'application des politiques réformatrices.

11 La consolidation du rôle de l'État comme État évaluateur et de l'évaluation comme axe structurant des politiques universitaires constituèrent les traits dominants de cette période. L'apparition de nouvelles maisons d'étude parut tendre vers une diversification graduelle de l'offre universitaire (bien que cela soit avec différentes fins selon les projets institutionnels) ainsi que vers la création de certaines niches de recherche scientifique. Dans cet environnement, à l'accroissement des inscriptions et des institutions s'ajoutèrent un développement et une diversification accélérés des troisièmes cycles universitaires.

\section{Héritages, revendications et tendances actuelles}

12 Ces dernières années, la carte politique des sociétés latinoaméricaines a connu des changements décisifs. Les gouvernements dits "progressistes " ou " post-néolibéraux " défendent l'idée d'une plus grande présence de l'État et formulent des critiques sévères sur les effets sociaux des politiques néolibérales. Du fait de son caractère dramatique et de sa gravité, la crise économique, politique et sociale qui frappa l'Argentine entre la fin de l'année 2001 et le début de 2002 constitua très probablement l'une des expressions les 
plus manifestes de ces mesures ${ }^{4}$. Dans l'ensemble de la région, parmi les propositions du gouvernement les moins en rupture, c'est en Argentine que s'est développé, au cours de ces sept dernières années, un vaste programme de politiques publiques. Il a impliqué un tournant important dans le rôle que prétend assumer l'État, ou, pour le moins, une prise de distance à l'égard des réformes appliquées dans les années 1990.

Dans le domaine de l'éducation, trois lois essentielles ont été promulguées: la Loi de financement de l'éducation, qui hausse le taux d'investissement pour le secteur de l'éducation, de la science et de la technologie à un palier de $6 \% \mathrm{du}$ PIB, la Loi d'éducation technique et professionnelle, qui s'efforce de rassembler et de mettre en ordre les modalités de l'éducation technique dans le secondaire. Enfin, en 2006, la promulgation de la Loi d'éducation nationale (LEN) abolit l'ancienne législation et rétablit le modèle de l'éducation primaire et secondaire. De ce fait, l'on a remis à l'ordre du jour l'idée traditionnelle de l'école secondaire comme niveau, après les résultats très controversés de l'application du cycle polymodal. L'on pourrait également souligner d'autres normes fixées par ces lois: la création d'un institut de formation pour les enseignants et la concrétisation de la demande historique de déclarer obligatoire l'achèvement du cycle de l'éducation secondaire et, enfin, le choix d'étendre les enseignements à la journée complète. Ceci eut pour conséquence l'extension du droit à l'éducation à treize années (une année de niveau initial, sept années d'éducation primaire et cinq de secondaire). Par ailleurs, la promotion récente du programme « allocation universelle par enfant » aurait permis une augmentation de près de $20 \%$ des inscriptions, l'assiduité scolaire constituant en effet l'une des obligations qui permet de percevoir l'aide sociale ${ }^{5}$.

Aux considérations antérieures, il convient d'ajouter que les commissions paritaires retrouvèrent toute leur place comme forme de régulation des discussions entre l'État et le corps enseignant, et que les salaires des maîtres et professeurs furent remis à niveau. Toutefois, il subsiste, dans la distribution des ressources éducatives nationales, un fédéralisme fiscal déséquilibré, si bien que l'investissement éducatif par élève et les salaires des enseignants révèlent de profondes disparités entre les différentes provinces (Rivas, 2004). Au cours de ces dernières années, plusieurs fonds nationaux ont été créés afin de corriger ces iniquités mais il demeure nécessaire d'inventer un apport salarial national qui se destine prioritairement aux enseignants des provinces qui ont le moins de ressources fiscales par habitant (ibid.).

15 Assurément, les politiques les plus actives de la part du ministère de l'Éducation ont pour objectif d'instaurer et de mettre en œuvre le caractère obligatoire de l'école secondaire. Il convient de signaler que l'universalisation de l'accès au niveau secondaire et les programmes de lutte contre le décrochage des nouveaux étudiants n'inversent pas, par eux-mêmes, les processus de sélection et de différenciation sociale : ceux-ci, en effet, continuent à se reproduire dans des circuits scolaires différenciés et parallèles et dans l'accès et la rétention au niveau tertiaire et quaternaire. Toutefois, cette politique contribue, dans une certaine mesure, à restituer aux secteurs les plus défavorisés des droits de citoyenneté bafoués. Dans cette perspective, il semble nécessaire de modifier certaines caractéristiques historiques du niveau, qui trahissent un fort caractère discriminatoire socialement et apparaissent comme un obstacle pour l'universalisation du droit. Nous faisons ici référence, en suivant Terigi (2008), à la classification très rigide des filières, avec des frontières strictement délimitées entre différents champs de connaissance, à la désignation concomitante des professeurs par spécialité, ainsi qu'à 
l'organisation du travail par heures de classe pour chaque discipline et non en poste scolaire, ceci se faisant au détriment direct de la concentration institutionnelle.

En ce qui concerne les politiques d'enseignement supérieur, un bilan provisoire des principales actions entreprises durant les sept dernières années doit commencer par signaler l'augmentation soutenue du budget universitaire. Cette injection de ressources constitue le moteur de mesures telles que la recomposition salariale des enseignants universitaires et pré-universitaires, la récente promulgation de la loi 26508/9 de départ à la retraite avec une pension de $82 \%$ du salaire - loi qui touche l'ensemble de la communauté des enseignants de l'université ${ }^{6}$-, la poursuite du programme d'aide à la recherche ainsi qu'une révision des normes orientées vers une amélioration de sa productivité. De la même manière, l'augmentation du budget des sciences et techniques et la création du ministère de la Science, de la Technologie et de l'Innovation sont des mesures qui traduisent également un progrès par rapport à l'époque antérieure. En tout état de cause, ces signes encourageants ne parviennent à constituer un nouveau programme politique mais s'apparentent plutôt à un processus qui navigue entre l'inertie antérieure et la recherche d'une orientation différente pour le secteur (Suasnábar, 2005). En suivant cette ligne, les politiques d'enseignement supérieur sembleraient accuser un retard certain si on les compare avec d'autres domaines de gestion marqués par le dynamisme, la capacité d'innovation politique et la vocation d'intervention et de régulation du pouvoir politique.

\section{Problèmes anciens et nouveaux dans la gouvernance de l'éducation}

17 Au cours de ces dernières années, le recouvrement progressif du rôle principal de l'État perceptible dans l'ensemble de lois promulguées et dans certains programmes spéciaux lancés par le ministère national - bien qu'il signifie un tournant décisif face aux politiques néolibérales des années quatre-vingt-dix, semble insuffisant pour relever avec succès les défis de la conjoncture actuelle. La priorité donnée à l'universalisation du niveau secondaire suppose d'inclure une part significative des jeunes, d'abaisser les taux de décrochage de la population qui assiste déjà aux enseignements de ce niveau et d'améliorer la qualité des apprentissages pour garantir un accès effectif à la connaissance. Présenté de la sorte, le défi actuel réside dans la capacité et la possibilité de bouleverser des dynamiques historiques qui ont caractérisé la matrice socio-éducative argentine. Sur ce point, les politiques éducatives récentes mettent en évidence la difficulté, afin de redéfinir des dispositifs capables de modifier le modèle sélectif qui traverse l'ensemble de la culture scolaire. Dans cette perspective, il paraît nécessaire de renforcer les capacités de gouvernance de l'éducation avec l'objectif de concevoir et de mettre en œuvre des politiques pédagogiques et curriculaires qui puissent accompagner les transformations actuelles mais également garantir le caractère soutenable du modèle de financement et sa juste redistribution entre les différentes provinces.

Dans le domaine de l'enseignement supérieur, l'absence d'un nouveau programme d'action politique est patente. La faiblesse des lignes directrices du gouvernement tend à renforcer et à concentrer les processus de prise de décision au sein des corps exécutifs de gouvernance des institutions, fait qui aggrave la dissociation entre une autonomie souhaitable et la nécessaire planification et coordination étatiques. Précisément, la 
difficulté à construire un nouveau sens (directionalité) pour le système d'enseignement supérieur dans son ensemble, loin de se limiter à une nécessité de planification stratégique ou de plan institutionnel des transformations, paraîtrait répondre aux intérêts conjoints de la gestion gouvernementale et de la corporation des recteurs. Dans ce contexte, la rupture de l'inertie politique pourrait remettre en jeu la gouvernabilité du secteur et ouvrir inutilement un nouveau front de tension sociale et politique ${ }^{7}$.

En somme, les débats de politique éducative et d'enseignement supérieur dans l'Argentine contemporaine témoignent d'héritages et de traditions contradictoires qui se traduisent, notamment, par une tension entre centralisation et décentralisation, régulation et autonomie, démocratisation et sélection, homogénéisation et reconnaissance des différences, diverses formes qui révèlent l'intensité des débats sociaux sur l'accès au savoir.

\section{BIBLIOGRAPHIE}

NDLR : on pourra consulter également Palamidessi M., « Argentine : une nouvelle loi nationale sur l'éducation », RIES n 44, 2007, p. 131-138 ; Palamidessi M., Galarza D., « Décentralisation et ruptures méthodologiques. Le cas de l'Argentine », RIES n² 29, 2002, p. 101-114.

AGIS E., CAÑETE C., PANIGO D. (2010) : El impacto de la asignación universal por hijo en Argentina. Buenos Aires: CEIL PIETTE. [en ligne]. [consulté le 22 mai 2010]. http://www.ceilpiette.gov.ar/ docpub/documentos/AUH_en_Argentina.pdf/

BRASLAVSKY C. et FILMUS D. (comp.) (1985) : Respuestas a la crisis educativa. Buenos Aires: Edit. Cantaro/FLACSO.

BRASLAVSKY C. (1985) : La discriminación educativa en la Argentina. Buenos Aires: FLACSO-GEL.

PUIGGRÓS A. (1990) : Sujetos, disciplina y curriculum en los orígenes del sistema educativo argentino. Buenos Aires: Edit. Galerna.

RIVAS A. (2004) : Gobernar la educación. Estudio comparado sobre el poder y la educación en la provincias argentinas. Buenos Aires: Granica.

ROSE N. (1997) : « El gobierno en las democracias liberales "avanzadas”: del liberalismo al neoliberalismo ». Revista Archipiélago. Cuadernos de crítica de la cultura n²9, Madrid.

SUASNÁBAR C. (2005) : « Entre la inercia y la búsqueda de una nueva agenda política: las políticas universitarias en el gobierno de Kirchner ». Temas y Debates. Revista Universitaria de Ciencias Sociales, $n^{\circ} 10$, año 9, 83-93.

TEDESCO J. C. (1986) : Educación y Sociedad en la Argentina (1880-1945). Buenos Aires: Edic. Solar.

TERIGI F. (2008) : « Los cambios en el formato de la escuela secundaria argentina: por qué son necesarios, por qué son tan difíciles ». Propuesta Educativa, n² 29, año 17, 63-71. 


\section{NOTES}

1. C'est précisément ce rôle hégémonique exercé par l'État national qui est à l'origine d'une grande partie des tensions à l'œuvre dans la gouvernance de l'éducation. Ainsi, le caractère très centralisé et homogénéisant apparut en contradiction avec le catactère fédéral proclamé de l'Argentine, puisque l'éducation - telle qu'elle a été définie par la Constitution nationale de 1853 - établit que l'enseignement primaire était sous la compétence des provinces. Cette intervention croissante fut légitimée vers 1905 par la promulgation de la loi Lainez, qui attribua à l'État national le droit de créer, soutenir et administrer des écoles dans les territoires provinciaux.

2. NdA: Quatre grandes étapes marquèrent l'évolution du débat sur la décentralisation. La première, dont les antécédents peuvent être identifiés dans les résolutions du Conseil national de l'éducation en 1956, s'exprima au début des années soixante sous la forme de conventions entre l'État national et les juridictions. Du fait des changements au sein du gouvernement national, seul le transfert d'un petit nombre d'écoles fut effectivement réalisé. Lors de la seconde étape, entre 1968 et 1970, le régime militaire de la « révolution argentine » poursuivit les accords de transfert avec quelques provinces et l'État national renonça en outre à créer de nouvelles écoles dans les territoires provinciaux. C'est à partir de 1978 que se produisit le transfert total des écoles primaires aux provinces, processus qui - contrairement aux précédents changements produisit de véritables traumatismes. Le nouveau gouvernement militaire imposa de manière drastique et unilatérale le transfert de quelque six mille écoles sans la moindre aide financière pour les juridictions. Finalement, en 1991, le second gouvernement constitutionnel qui suivit le retour de la démocratie lança la quatrième étape de la décentralisation, qui acheva le transfert de la totalité des collèges moyens et lycées tertiaires nationaux aux provinces. NDLR : voir aussi Aguerrondo I. (2003), «Décentraliser pour centraliser : le renforcement du ministère national pour développer l'organisation fédérale en Argentine ", Revue internationale d'éducation de Sèvres $\mathrm{n}$ - 32, p. 29-41.

3. Tels furent le cas de nouveaux organismes créés comme la Commission nationale d'évaluation et d'accréditation universitaire (CONEAU), le Conseil des universités et les conseils de planification régionale de l'enseignement supérieur (CPRES) que le secrétariat des Politiques universitaires « chargea » de l'évaluation des institutions et des filières de premier, deuxième et troisième cycles ainsi que de l'accréditation qui leur est liée.

4. Il convient de rappeler que, comme corollaire de la crise, l'on enregistra en 2002 une chute, d'une année sur l'autre, de 10,9 \% du PIB, avec un taux de chômage de la population active de $21,5 \%$, soit 3,4 millions de personnes pour l'ensemble de la population. En octobre 2002, 27,5\% des citoyens furent en situation d'indigence et $57,5 \%$ de pauvreté.

5. À la fin de l'année 2009, le gouvernement de Cristina Fernández de Kirchner a lancé le programme "allocation universelle par enfant » (AUH), destiné aux travailleurs sans emploi et aux employés précaires. Elle leur permet de percevoir une somme de 180 pesos par mois (environ 37 euros) pour chaque enfant âgé de moins de 18 ans (à concurrence de cinq enfants par famille) et à vie pour ceux qui souffrent d'un handicap. Ce programme a permis de faire sortir de la pauvreté entre 1,4 et 1,8 million de personnes. Entre 700000 et 1,1 million d'entre elles ont 18 ans et moins. En chiffres absolus, le taux de pauvreté a chuté, au cours du second semestre de l'année 2009 , de $14,1 \%$ à 9,5\% après la mise en place de l'AUH. Par la suite, entre 1 et 1,5 million de personnes ont abandonné la condition d'« indigent ». Parmi eux, entre 680000 et 1,03 million de personnes ont 18 ans ou moins (Agis, Cañete, Panigo, 2010).

6. Grâce au cadre fixé par cette loi, les universitaires retraités obtiendraient un alignement automatique de leurs pensions - en conservant la proportion de $82 \%$ par rapport au salaire de la 
charge qu'ils occupaient au moment de leur retraite - sur les augmentations des salaires des enseignants en activité.

7. À cet égard, il n'est pas hasardeux qu'une partie du débat politique universitaire autour de la promulgation d'une nouvelle loi d'enseignement supérieur ait débouché davantage sur des actions et mesures « correctives » des dispositifs mis en œuvre que sur la définition de nouveaux axes d'orientation pour les politiques.

INDEX

Index géographique : Argentine

Mots-clés : gouvernance, réforme de l'enseignement, système scolaire

\section{AUTEURS}

\section{CLAUDIO SUASNÁBAR}

Claudio Suasnábar est docteur en sciences sociales et titulaire d'un magistère en éducation de la Facultad Latinoamericana de Ciencias Sociales de Buenos Aires, Argentine. Professeur de politique et législation éducatives et d'histoire et politique du système éducatif à l'Université nationale de La Plata, il est aussi le coordinateur de l'Observatorio Sindical de Políticas Universitarias del Instituto de Estudios y Capacitación de la fédération nationale des professeurs des universités.

\section{LAURA INÉS ROVELLI}

Laura Inés Rovelli est titulaire d'un magistère en sciences sociales avec une spécialisation en éducation de la Facultad Latinoamericana de Ciencias Sociales de Buenos Aires, où elle est doctorante. Boursière du Consejo Nacional de Investigaciones Científicas y Tecnológicas, elle enseigne à l'Université nationale de La Plata. 